Western University Scholarship@Western

Centre for Decision Sciences and Econometrics

Technical Reports

Economics Working Papers Archive

1987

\title{
The Econometric Analysis of Models with Risk Terms
}

\author{
A. R. Pagan \\ Aman Ullah
}

Follow this and additional works at: https://ir.lib.uwo.ca/economicscdse_tr Part of the Economics Commons

\section{Citation of this paper:}

Pagan, A. R., Aman Ullah. "The Econometric Analysis of Models with Risk Terms." Centre for Decision Sciences and Econometrics Technical Reports, 16. London, ON: Department of Economics, University of Western Ontario (1987). 


\title{
THE CENTRE FOR DECISION SCIENCES AND ECONOMETRICS
}

The Econometric Analysis of Models with Risk Terms

\author{
Adrian Pagan \\ and \\ Aman Ullah
}

TECHNICAL REPORT NO. 16

JULY 1987

is

Centre For Decision Sciences And Econometrics

Social Science Centre

The University of Western Ontario

London, Ontario N6A 5C2

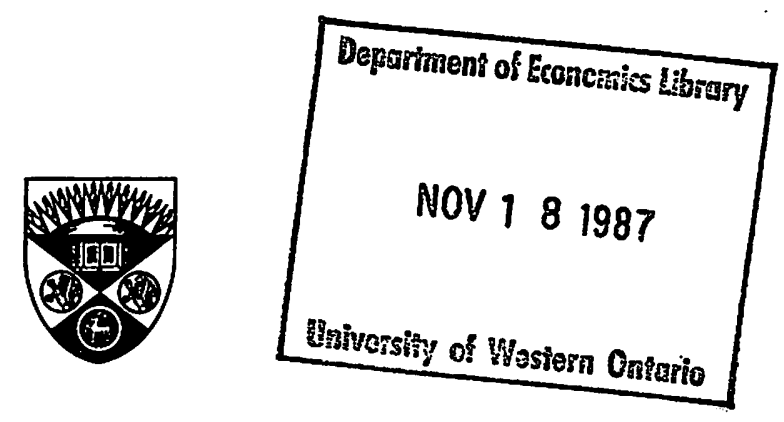


The Econometric Analysis of Models with Risk Terms*

\section{Adrian Pagan}

The University of Rochester. Rochester, N.Y., U.S.A. 14627

Aman Ullah

The University of Western Ontario, London. Ontario, Canada

April, 1986, Revised February, 1987.

* He are grateful to Hashem Pesaran for his comments upon this paper.

Deparimeat of Exoscmiss tharary

NOV $18198 ?$

Universiny ct Westen Ontario 


\section{Introduction}

That the world inhabited by economic agents is a risky one has long been accepted and emphasized by economic theorists. In quantitative models. however, much less importance has traditionally been accorded to such effects. The rational expectations revolution in econometric analysis was largely concerned with how to model the mean of a random variable conditional upon an information set, and only minimal attention was paid to second and higher moments of the corresponding conditional distribution. Even in those instances where higher order moments entered the analysis, as in the Capital Asset Pricing Model, portfolio models, or the first order conditions from many "Euler equation" models, they were typically assumed constant, and so the effects of risk factors became absorbed into the parameter set. Frankel (1955) is a good illustration of this point.

Al though the above seems a fair description of much research, events in the 1970's conspired to interest the applied economist in the difficulties of accounting for changing risk. The initial impetus seems to have come from a rise in inflation rates, and a group of studies was spawned that sought to examine the economic effects of greater unpredictability in either the levels of inflation or relative prices, for example Klein (1977) and Hercowitz (1981). Furthermore, as the 1970's wore on, freer exchange rates and much more flexible monetary policies in many economies meant that some allowance might need to be made for what was perceived as an increasingly risky environment. 
There now exist a fair number of studies attempting to allow for a changing risk term in economic models; a very small sample would have to mention Vanderhoff (1983), Mascaro and Meltzer (1983), Lawrence (1983), Engle (1983) and Gylfasson (1981). It is noticeable, however, that there are few theoretical papers on which econometric methods should be employed in this context, and there has been considerable diversity in the way in which each of the researchers has approached the problem. In an earlier paper. Pagan (1984), one of us briefly looked at the area, concluding that at least one of the popular methods was unlikely to be satisfactory, and suggesting some alternatives. However, the treatment was not very comprehensive, and it therefore seems appropriate to re-examine the issues here in somewhat greater depth than before.

Section 2 of this paper considers the estimation of a linear model containing a term representing the risk originating from a failure to perfectly predict some variable. Theoretical models show that this risk is related to the moments of the probability density function of the variable conditioned upon whatever information agents use in their optimization. For expository purposes we assume that the conditional variance is the appropriate moment, propose that it be replaced by functions of the observed data, and then recommend estimation of unknown parameters by instrumental variables, instruments being constructed from the information set. Conditions are set out under which the proposed estimator is consistent and in which correct inferences can be made. Section 3 of the paper looks at issues arising when the level of risk is to be explained rather than when it is an explanator. 
Within the literature a variety of proxies for risk can be found.

Section 4 looks at these to see how satisfactory each method is when used as a measure of risk in a modeling environment. In general the proxies suffer from a variant of the "errors in variables" problem, and therefore should only be used in conjunction with an instrumental variables estimator: if substituted directly into a regression an underestimate of the effect of risk on decisions is likely. An exception to this rule occurs if a parametric model for the risk term is adopted, but this methodology has its own problems involving potential mis-specification, which can be partially alleviated by following our instrumental variables approach. Finally. section 5 applies some of the ideas in the paper.

\section{Risk as a Regressor}

\subsection{An Instrumental Variables Estimator}

Many macro-economic models are formulated by having agents optimally choose a variable $y_{t}$ on the basis of some information set $\mathscr{F}_{t}$. It is assumed here that this optimization yields a linear decision rule of the form

$$
E\left(y_{t} \mid F_{t}\right)=\bar{x}_{t} \gamma+\sigma_{t}^{2} \delta \quad t=1.2, \ldots, T
$$

where $\bar{x}_{t} \in \mathcal{F}_{t}$ is a (1xs) vector and $\sigma_{t}^{2}$ is the variance of some variable $\psi_{t}$ conditional upon ${ }_{t} \cdot \sigma_{t}^{2}$ represents a risk term arising from the failure of agents to be able to correctly predict the variable $\psi_{t}$. A model such as (1) can arise in a number of ways. Standard mean/variance analysis produces it. 
but there exist more general models in which $\sigma_{t}^{2}$ can be taken as a linear approximation to the expectation of a non-linear function of a random variable, and this is then interpreted as risk, e.g. Leland (1961) for consumption. Each of $\mathscr{F}_{t}, \Psi_{t}$, and $\bar{x}_{t}$ need to be defined by the theoretical context: in Stockman (1978) for example, $\Psi_{t}$ is a vector of variables involving the domestic and foreign money supplies and the real rate of return, while $\mathcal{F}_{t}$ will be whatever information an agent is assumed to possess in the optimizing exercise. It is convenient to treat $\sigma_{t}^{2}$ as a scalar, as the extension to the vector case is obvious. Moreover, although it will be the second moment $\sigma_{t}^{2}$ which is the focus of this paper, the methods advanced clearly extend to any moment of the density of $\psi_{t}$ conditional upon $\mathscr{F}_{t}$. or even the covariance between two variables (say) $w_{1 t}$ and $w_{2 t}$.

(1) may be converted to the form

$$
y_{t}=\bar{x}_{t} \gamma+\sigma_{t}^{2} \delta+e_{t}
$$

where $e_{t}$ is an error term with the property $E\left(e_{t} \mid F_{t}\right)=0$. The estimation problem is that no series on $\sigma_{t}^{2}$ exists, and theory does not describe exactly how $\sigma_{t}^{2}$ varies with $F_{t}$. In this paper we will address the various solutions that have been proposed to this dilemna. First, however, let us suppose that it was possible to construct a series $\phi_{t}$ such that $\phi_{t}^{2}$ was a function of some index $N$ (that does not depend on $t$ ) and that $\phi_{t}^{2}(N) \stackrel{\text { a.s. }}{\longrightarrow} \sigma_{t}^{2} \forall t$ as $N \rightarrow \infty$. Such a series will be said to possess a "strong property". since for large enough $N$ it is possible to regard $\phi_{t}^{2}$ as $\sigma_{t}^{2}$. By contrast it may be that the sole series $\phi_{t}$ which is available possesses only a "weak property", $E\left(\phi_{t}^{2} \mid F_{t}\right)=$ $\sigma_{\mathrm{t}}^{2}$ 
As noted above, if $\phi_{t}^{2}$ had the strong property it would be sensible to replace $\sigma_{t}^{2}$ in (2) with $\phi_{t}^{2}$ and to regress $y_{t}$ against $x_{t}$ and $\phi_{t}^{2}$ to obtain an estimate of $\delta$. It is tempting to do the same thing when it has the weak property, but then the model (2) becomes

$$
y_{t}=\bar{x}_{t} \gamma+\phi_{t}^{2} \delta+\epsilon_{t}=\bar{x}_{t} \gamma+\phi_{t}^{2} \delta+e_{t}+\delta\left(\sigma_{t}^{2}-\phi_{t}^{2}\right)
$$

Al though $E\left(\bar{x}_{t} e_{t}\right)=0$ and it may not be unreasonable to assert that $E\left(\phi_{t}^{2} e_{t}\right)=$ 0 , it is clear that $E\left(\phi_{t}^{2} \epsilon_{t}\right)$ will not be zero as it involves $\delta\left(E\left(\sigma_{t}^{4}\right)-E\left(\phi_{t}^{4}\right)\right)$, which is only zero in degenerate cases. When $\phi_{t}$ is normal and $F_{t}$ consists of non-stochastic elements, $\delta\left(E\left(\sigma_{t}^{4}\right)-E\left(\phi_{t}^{4}\right)\right)$ is $-2 \sigma_{t}^{4}$. Pagan (1984, p234) notes that the size of the inconsistency in the OLS estimator of $\delta$ can therefore be quite large. It should be observed that no such problems arise if $\phi_{t}$ had the strong property since $\delta\left(\sigma_{t}^{2}-\phi_{t}^{2}\right) \stackrel{\text { a.s. }}{\longrightarrow} 0$ as $N \longrightarrow \infty$.

Section 4 of the paper discusses the selections of $\phi_{t}$ that have been made in the literature. With one exception the proxies for $\sigma_{t}^{2}$ that have been suggested possess only a weak property (at best). making their use in a regression context suspect. But there is a way out of the difficulty. As the analysis above shows, the inconsistency in the OLS estimator arises from the fact that the "true" regressor $\sigma_{t}^{2}$ is observed with error, and so ols suffers from an errors in variables bias. A standard solution to this is to estimate (3) not by OLS but by instrumental variables (IV). From the orthogonality condition $E\left(e_{t}+\delta\left(\sigma_{t}^{2}-\phi_{t}^{2}\right) \mid F_{t}\right)=0$, we assume that there exist $p=s+1$ 
instruments $z_{t}$ constructed from $\mathscr{F}_{t} \cdot{ }^{1}$ Having done so restrictions need to be placed upon the nature of $x_{t}=\left(\bar{x}_{t} \phi_{t}^{2}\right), z_{t}$ and $\epsilon_{t}$ to get desirable asymptotic properties for the IV estimator. It is convenient here to use the terminology and results from the asymptotic theory of mixing processes and we extensively utilize results from thite (1984). Definitions and explanations of the terms can be found there.

\section{ASSUMPTIONS}

$$
\left\{\left(z_{t}, x_{t}, \epsilon_{t}\right)\right\} \text { is either a phi (or alpha) mixing sequence with mixing }
$$
coefficients of size $r^{\circ} /\left(r^{\circ}-1\right), r^{\prime}>1$ (or of size $r^{\circ} /\left(r^{\prime}-1\right), r^{\prime}$ >1). where $r^{\prime}=r+a$ for some $r \geq 1$ and $0<a \leq r$;

(a) $E\left(z_{t} \epsilon_{t}\right)=0$

(b) $E\left|z_{t i} \epsilon_{t}\right|^{2 r^{\prime}}<\Delta<\infty$ for $r^{\prime}>1, i=1, \ldots$ and all $t$

(c) $V_{b T}=\operatorname{var}\left(T^{-1 / 2} \sum_{t=b+1}^{b+T} z_{t} \epsilon_{t}\right) ; V_{T}=V_{O T}$ and $\exists v$ finite and positive definite such that $V_{b T}-V \rightarrow 0$ as $T \rightarrow \infty$ uniformly in $b$;

(a) $E\left|z_{t i} x_{t j}\right|^{r^{\prime}}<\Delta_{1}<\infty$ for $r^{\prime}>1$ and all $i=1, \ldots$ p: $j=1, \ldots, p ; t=1, \ldots, T$;

(b) $Q_{T}=E(Z \cdot X / T)$ has uniformly full column rank

(c) $\hat{\mathrm{P}}_{\mathrm{T}}-\mathrm{P}_{\mathrm{T}} \stackrel{\mathrm{p}}{\rightarrow} 0$ where $\left\{\mathrm{P}_{\mathrm{T}}\right\}$ represents a $\mathrm{p} \times \mathrm{p}$ weighting matrix for the $p$ instruments and is $O(1)$ and uniformly positive definite.

${ }^{1}$ If more instruments are available than $p$, generalized instrumental variable (Sargan (1958)) or GM estimators (Hansen (1982)) will need to replace the simple IV procedure adopted here. 
We have chosen to state the conditions in terms of the joint vector $\left(z_{t}, x_{t}, \epsilon_{t}\right)$ rather than $\left(z_{t}, x_{t}, e_{t}\right)$, as it saves a good deal of notation. However, since $\phi_{t}^{2}-\sigma_{t}^{2}$ will also mix as in (i), and $\epsilon_{t}$ is the sum of $e_{t}$ and $\delta\left(\phi_{t}^{2}-\sigma_{t}^{2}\right)$. it is clear that no generality is lost in doing this.

We can immediately provide the following proposition.

Proposition 1: If (3) is written as $y_{t}=x_{t} \beta+\epsilon_{t}$ or $y=x \beta+\epsilon$, and $p=s+1$, under assumptions $(i)$ - (iii) the instrumental variable estimator of $\beta, \tilde{\beta}=$ $\left(Z^{\prime} X\right)^{-1} Z^{\prime} y$, is a consistent estimator of $\beta$, while $D_{T}^{-1 / 2} T^{1 / 2}(\tilde{\beta}-\beta) \stackrel{d}{\rightarrow} N\left(0, I_{p}\right)$, where $D_{T}=Q_{T}^{\prime} V_{T}^{Q_{T}}$.

Proof: Theorem 5-22 of White $(1984, \mathrm{p} 126)$ with $\mathrm{P}_{\mathrm{T}}=\left(\mathrm{Q}_{\mathrm{T}}^{\prime}\right)^{-1}$.

How restrictive is Proposition 1? Loosely, the assumptions restrict the processes $x_{t}, \phi_{t}^{2}$ and $e_{t}$ to be stationary; some non-stationarity in the second moment is possible but it is severely circumscribed. Now, since $\sigma_{t}^{2}=E\left(\left(\psi_{t}-\right.\right.$ $E\left(\left(\psi_{t} \mid F_{t}\right)^{2} \mid F_{t}\right)$, it is clear that $\phi_{t}=\psi_{t}-E\left(\psi_{t} \mid F_{t}\right)$, that is it is the unanticipated part of $\psi_{t}$. Since we would expect that $\psi_{t}$ and $E\left(\psi_{t} \mid F_{t}\right)$ would be co-integrated, using Engle and Granger's (1987) term, the unanticipated quantity should therefore be a stationary process. It also seems reasonable that the correlation of $\phi_{t}$ with values in the past should die out fairly quickly the further back one goes.

However, we cannot be so sanguine about other variables that drive the conditional mean from any optimizing exercise resulting in (1). As observed 
earlier. Stockman's (1978) model has $y_{t}$ being the spot rate and $\bar{x}_{t}$ the forward rate, and both of these processes are commonly regarded as ARIMA rather than ARMA processes. A variety of responses can be made to this challenge. First, the optimizing theory may suggest that the non-stationary variables are co-integrated, i.e. there exists a linear combination of them which is stationary. That is the case in Stockman's formulation; the coefficient $r$ on the forward rate is unity so that (2) could be written as $y_{t}-\bar{x}_{t}=\sigma_{t}^{2} \delta+e_{t}$ : a simple re-definition of $y_{t}$ as $y_{t}-\bar{x}_{t}$ then allows the assumptions to be invoked. More generally, $y_{t}$ can be thought of as a linear combination of all the co-integrated variables in the optimizing exercise, while $\bar{x}_{t}$ contains only those members of $F_{t}$ that are stationary. When the co-integrating vector is not known, it can be estimated by regressing the co-integrated variables upon one another, with the residuals from this regression becoming $y_{t}-$ this is the "two-step" procedure in Engle and Granger (1987) and the reader is referred there to the proof that replacing the co-integrating parameters by estimates does not affect the limiting distribution of the estimators of parameters associated with stationary variables.

However, it is not in fact necessary to regress out all the non-stationary variables. Suppose that $(3)$ is re-parameterized so that $\bar{x}_{t}$ is separated into $\bar{x}_{1 t}$ (non-stationary) and $\bar{x}_{2 t}$ (stationary) variables. The $r$ vector is partitioned conformably into $\gamma^{\prime}=\left(\gamma_{1}^{\prime} \gamma_{2}^{\prime}\right)$. while $\beta_{2}^{\prime}=\left(\gamma_{2} \delta\right)$ and $\beta_{1}$ $=\gamma_{1}$. Let $\bar{x}_{1 t}$ be its own instrument and denote the instruments for $\bar{x}_{2 t}$ and $\phi_{t}^{2}$ as $z_{1 t}$. Then, if $\bar{x}_{1 t}$ is ARIMA $(0 . g .0)$ while $\left(\bar{x}_{2 t}, z_{1 t} \cdot \phi_{t}^{2}\right)$ are strictly stationary processes, Lemma 2 of Sims. Stock and Watson (1986) shows that cov $\left(T^{g}\left(\tilde{\beta}_{1}-\beta_{1}\right) \cdot T^{1 / 2}\left(\tilde{\beta}_{2}-\beta_{2}\right)\right)$ is zero, so that the limiting normal distribution in Proposition 1 applies to the estimators of the parameters of the stationary variables. For $\tilde{\beta}_{1}$, the limiting distribution may or may not be 
normal depending upon whether there is drift in the ARIMA process or not. Because of this separability result, estimation of $\delta$ can proceed as if $y_{t}$ and $\bar{x}_{t}$ were stationary, and hence Proposition 1 and its extensions given below apply directly.

Proposition 1 shows that inference about $\beta$ cannot be based upon the standard covariance matrix for $T^{1 / 2}(\tilde{\beta}-\beta)$ provided by most instrumental variables computer prograns. That estimator is $\sigma^{2}\left(\mathrm{TQ}_{\mathrm{T}}\right)^{-1} \mathrm{~T}^{-1} \mathrm{Z} Z\left(\mathrm{TQ} \mathrm{Q}_{\mathrm{T}}\right)^{-1}$, where $\sigma^{2}$ is an estimate of the variance of $\epsilon_{t}$, and, even if $\epsilon_{t}$ were independently distributed, does not equal $V_{T}$ asymptotically owing to heteroskedasticity in the errors $\epsilon_{t}$. It is necessary therefore to provide an estimator of the covariance matrix of $T^{1 / 2}(\tilde{\beta}-\beta)$ which will be robust to the general form of evolutionary behaviour assumed for $\phi_{t}$. What is required is that $V_{T}$ be consistently estimated, and for this we have the following proposition.

Proposition 2: Under the assumptions earlier but with mixing coefficients of size 2 (or of size $\left.2(r+a-1)^{-1}(r+a), r>1\right)$, and $r^{\prime}=2(r+b)$ for some $b>0$ in (ii)(b) and $(i i i)(a)$, the estimator

$$
\hat{v}_{T}=T^{-1} \sum_{t=1}^{T} z_{t}^{\prime} z_{t} \tilde{\epsilon}_{t}^{2}+T^{-1} \sum_{T=1}^{\ell} \sum_{t=T+1}^{T}\left(z_{t}^{\prime} \tilde{\epsilon}_{t} \tilde{\epsilon}_{t-T} z_{t-T}+z_{t-T}^{\prime} \tilde{\epsilon}_{t-T} \tilde{\epsilon}_{t} z_{t}\right)
$$

is a consistent estimator of $V_{T}$ when $\tilde{\epsilon}_{t}=y_{t}-x_{t} \tilde{\beta}$ and $e$ is $o\left(T^{1 / 4}\right)^{2}$.

\footnotetext{
2Newey and West (1986) give a weighted version which is a consistent estimator but ensures that $\hat{V}_{T}$ would be positive definite.
} 
Proof: White (1984. Theorem 6.20), except the restriction upon $\ell$ is the correction found in Phillips (1985) and Newey and West (1986).

Consistent estimation of $\delta$ when a $\phi_{t}^{2}$ is available such that $E\left(\phi_{t}^{2} \mid F_{t}\right)=\sigma_{t}^{2}$ is therefore easy, and Propositions 1 and 2 give the limiting theory. Unfortunately, since $\phi_{t}=\psi_{t}-E\left(\psi_{t} \mid F_{t}\right)$, and it is going to be rare that $E\left(\psi_{t} \mid F_{t}\right)$ is available (or known). $\phi_{t}$ will need to be estimated from available data. In most econometric work $E\left(\psi_{t} \mid F_{t}\right)$ is made a linear function of $q$ elements $\left(w_{t}\right)$ taken from $F_{t}$. that is $\psi_{t}=E\left(\psi_{t} \mid F_{t}\right)+\phi_{t}$ is assumed to be $\psi_{t}=$ ${ }{ }_{t} \theta_{0}+\phi_{t}$. where $\theta_{0}$ is the true value of some parameters. $\phi_{t}$ would then be estimated by replacing $\theta_{0}$ by some estimate $\hat{\theta}$.

Proposition 3 is concerned with the properties of the instrumental variables estimates when $\phi_{t}^{2}$ is replaced by $\hat{\phi}_{t}^{2}$ and $\hat{\phi}_{t}=\psi_{t}-w_{t} \hat{\theta}$. It may be possible to state the conditions needed for Proposition 3 to hold in terms of the more primitive assumptions made earlier for Proposition 1, but it is easier to impose conditions directly upon $w_{t}$. Then Proposition 3 states that the limiting distribution of the "feasible" IV estimator $T^{1 / 2}(\hat{\beta}-\beta), \hat{\beta}=$ $(Z \cdot \hat{x})^{-1} z \cdot y$, where $\hat{x}$ has $t^{\text {th }}$ row $\left(\bar{x}_{t} \hat{\phi}_{t}^{2}\right)$, is the same as that of $T^{1 / 2}(\tilde{\beta}-\beta)$.

Proposition 3: If (i) $\hat{\theta}-\theta_{0}$ is $0_{p}\left(T^{-1 / 2}\right)$. (ii) the set of conditions given earlier for Proposition 1 hold but with $\left(z_{t}^{\prime}, x_{t}^{0}, \epsilon_{t}\right)$ augmented with $w_{t} \cdot$ (iii)

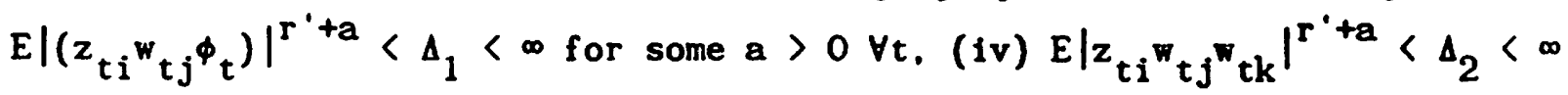


for some $a>0 \forall t, i=1, \ldots, p, j=1, \ldots, q$

$$
T^{1 / 2}(\tilde{\beta}-\beta)-T^{1 / 2}(\hat{\beta}-\beta) \text { is o } p(1)
$$

Proof: As $\tilde{\beta}=\left(Z^{\prime} X\right)^{-1} Z^{\prime} y$ and $\hat{\beta}=\left(Z^{\prime} \hat{X}\right)^{-1} Z^{\prime} y$ we first need to show that $T^{-1} Z^{\prime} X$ $-T^{-1} Z \cdot \hat{X}$ is $o_{p}(1)$.

$$
T^{-1} Z \cdot X-T^{-1} Z \cdot \hat{X}=T^{-1} Z \cdot(X-\hat{X})=\left[\begin{array}{c}
0 \\
T^{-1} \Sigma z_{t}^{\prime}\left(\phi_{t}^{2}-\hat{\phi}_{t}^{2}\right)
\end{array}\right]
$$

The $i^{\text {th }}$ element in $T^{-1} \Sigma z_{t}^{\prime}\left(\phi_{t}^{2}-\hat{\phi}_{t}^{2}\right)$ is

$$
T^{-1} \Sigma z_{i t}\left(\phi_{t}^{2}-\hat{\phi}_{t}^{2}\right)=2\left(T^{-1} \Sigma z_{i t}{ }^{w}{ }_{t}{ }_{t}\right)\left(\hat{\theta}-\theta_{0}\right)-\left(\hat{\theta}-\theta_{0}\right) \cdot\left(T^{-1} \Sigma z_{i t} w_{t}{ }^{w}{ }_{t}\right)\left(\hat{\theta}-\theta_{0}\right) .
$$

By Proposition 3.50 of White (1984), $\left(z_{i t} w_{t} \phi_{t}\right)$ is a mixing sequence of order $\left(r^{\prime}-1\right)^{-1} r^{\prime}$, while by Theorem 3.49 of thite $z_{i t} w^{\prime} t^{w} t$ also mixes. From the strong law of large numbers for mixing processes - White, Corollary 3.48 and conditions $(i i)$ and $(i i i)$ of the proposition, $T^{-1} \Sigma z_{i t} t_{t} \phi_{t}$ and $T^{-1} \sum z_{i t}{ }^{w} t^{w} t$ both converge to their expectations. Hence (5) is $o_{p}(1)$ and

$$
\tilde{\beta}-\hat{\beta}=\left(T^{-1} Z \cdot X\right)^{-1} T^{-1 / 2} \Sigma z_{t}^{\prime}\left(\phi_{t}^{2}-\hat{\phi}_{t}^{2}\right) \delta+o_{p}(1) .
$$

Inspection of (5) multiplied by $T^{1 / 2}$ shows that with condition ( $i$ ) and $T^{-1} \Sigma z_{i t} w_{t} \phi_{t} \stackrel{p}{\rightarrow} 0$ (because $\left.E\left(\phi_{t} \mid z_{i t} \cdot w_{t}\right)=0\right), T^{-1 / 2} \Sigma z_{t}^{\prime}\left(\phi_{t}^{2}-\hat{\phi}_{t}^{2}\right)$ is o ${ }_{p}(1)$. verifying the proposition. 


\subsection{Problems in Constructing Proxies and Instruments}

An emphasis on the IV method for handling the errors in variables problem arising when $\phi_{t}^{2}$ replaces $\sigma_{t}^{2}$ fits well with the recent tendency in macroeconomic research to generate estimators from or thogonality conditions. The popularity of this strategy is partly explained by the robustness of the IV estimator to any failure of the econometrician to have the complete information set available to the agent. Thus if (1) $\operatorname{was} E\left(y_{t} \mid F_{t}\right)=E\left(\psi_{t} \mid F_{t}\right) \gamma$. and the only information available to an econometrician was $\mathscr{G}_{t} C_{t}$, an estimable equation is

$$
y_{t}=E\left(\psi_{t} \mid \varphi_{t}\right) \gamma+e_{t}+\left(E\left(\psi_{t} \mid \mathscr{F}_{t}\right)-E\left(\psi_{t} \mid \varphi_{t}\right)\right) \gamma
$$

If IV is applied to (7) with instruments chosen from $\mathscr{\varphi}_{t}$, the estimator of $\gamma$ is consistent as $\left.E\left(E\left(\psi_{t} \mid F_{t}\right)-E\left(\psi_{t} \mid \mathscr{s}_{t}\right)\right) \mid \mathscr{\xi}_{t}\right)=0$ from the law of iterated expectations (Nelson (1975), Wickens (1982)). Unhappily, such a felicitous outcome does not carry over to the estimation of the effects of risk. Proposition 4 deals with this point.

Proposition 4: Unless $\mathscr{E}_{\mathrm{t}}=\mathcal{F}_{\mathrm{t}}$, variables in $\mathscr{\varphi}_{\mathrm{t}}$ will generally be correlated with the error term in any model in which $\sigma_{t}^{2}=E\left(\psi_{t}^{2} \mid g_{t}\right)$ is replaced by $\bar{\sigma}_{t}^{2}=$ $E\left(\psi_{t}^{2} \mid \varepsilon_{t}\right)$

Proof: Let $\bar{\phi}_{t}=\psi_{t}-E\left(\psi_{t} \mid \xi_{t}\right)$ so that $\sigma_{t}^{2}=E\left(\phi_{t}^{2} \mid g_{t}\right), \bar{\sigma}_{t}^{2}=E\left(\bar{\phi}_{t}^{2} \mid \phi_{t}\right)$ and the model estimated by the investigator is based upon

$$
y_{t}=\bar{x}_{t} \gamma+\bar{\sigma}_{t}^{2} \delta+e_{t}+\left(\sigma_{t}^{2}-\bar{\sigma}_{t}^{2}\right) \delta
$$


Evaluating the expectation of the last term in (8) conditional upon $y_{t}$ gives $\delta E\left(\left(\sigma_{t}^{2}-\bar{\sigma}_{t}^{2}\right) \mid \xi_{t}\right)=\delta E\left[\left(E\left(\phi_{t}^{2} \mid \mathscr{F}_{t}\right)-E\left(\bar{\phi}_{t}^{2} \mid \varepsilon_{t}\right)\right) \mid \varepsilon_{t}\right]$, substituting $\phi_{t}=\bar{\phi}_{t}+$ $E\left(\psi_{t} \mid \varphi_{t}\right)-E\left(\psi_{t} \mid F_{t}\right)$, and simplifying using the law of iterated expectations shows that $\delta E\left(\left(\sigma_{t}^{2}-\sigma_{t}^{2}\right) \mid \mathscr{\varphi}_{t}\right)=\delta\left\{\left[E\left(\psi_{t} \mid \mathscr{\varphi}_{t}\right)\right]^{2}-E\left[\left(E\left(\psi_{t} \mid \mathscr{F}_{t}\right)\right)^{2} \mid \mathscr{\xi}_{t}\right]\right\}$. This expression is generally non-zero as Jensen's inequality for conditional expectations has $E\left[\left(E\left(\psi_{t} \mid F_{t}\right)\right)^{2} \mid \varphi_{t}\right] \geq\left[E\left(\psi_{t} \mid \varphi_{t}\right)\right]^{2}$.

Because of the correlation of members of the set $\varphi_{t}$ with $\left(\sigma_{t}^{2}-\bar{\sigma}_{t}^{2}\right)$. consistent estimation of $\delta$ will almost always require that $g_{t}$ be known. Only if $\delta=0$, in which case $\delta\left(\sigma_{t}^{2}-\bar{\sigma}_{t}^{2}\right)$ does not appear in the error term of (8), will consistent estimation be possible with a truncated information set. This makes it crucial that $\mathscr{G}_{t}$ be set wide enough to encompass $\mathscr{F}_{t}$, when generating the conditional expectation. Such a lack of robustness makes the estimation of models with risk terms a very difficult task. Throughout the remainder of this paper we maintain the assumption that $\mathscr{E}_{\mathrm{t}}=\mathscr{F}_{\mathrm{t}}$.

Even if the set of variables in $\mathcal{F}_{t}$ can be identified however, the assumption that $E\left(\psi_{t} \mid F_{t}\right)$ is linear in $w_{t}$ could well be incorrect. Moreover. no guidance has been given concerning the selection of instruments $z_{t}$. It is likely that $z_{t}$ will have to be a non-linear function of $\mathscr{F}_{t}$. Suppose that $E\left(\psi_{t} \mid F_{t}\right)=\theta \psi_{t-1}$ and that $\phi_{t}$ was normal and identically distributed with zero mean and conditional variance $\sigma_{t}^{2}=\alpha_{0}+\alpha_{1} \phi_{t-1}^{2}$. Because $\psi_{t-1} \in \mathcal{F}_{t}$. it might be taken as an instrument for $\phi_{t}^{2}$, but clearly $E\left(\psi_{t-1} \phi_{t}^{2}\right)=0$ when $|\theta|<1$. violating the requirement for a good instrument in assumption (iii)(b). If, however, $\psi_{t-1}^{2}$ 
is taken as the instrument, $E\left(\psi_{t-1}^{2} \phi_{t}^{2}\right) \neq 0$ and it satisfies the conditions. Of course an ideal instrument for $\phi_{t}^{2}$ would be $\sigma_{t}^{2}$. but economic theory is not of much help in indicating how $\sigma_{t}^{2}$ can be expected to vary with $F_{t}$ - all models that allow for risk merely note the dependence of decisions upon the conditional variance, assuming that agents will learn about how the available information maps into this moment.

How then could estimates of the conditional mean and variance of $\psi_{t}$ be found that do not rely on linearity? Our objective is to estimate $E\left(g\left(\psi_{t}\right) \mid w_{t}\right)$, where $w_{t}$ represents the basic elements in ${ }_{t}$ and $g\left(\psi_{t}\right)$ is either $\psi_{t}$ or $\psi_{t}^{2}$. Non-parametric estimation procedures have been advanced in recent years to estimate such conditional moments, with the main econometric references being Bierens (1985), Robinson (1983), Robinson (1986), and

Ullah (1986). Readers are referred to these papers for a more detailed discussion of the methods employed.

Robinson (1983) is perhaps the most general treatment of those mentioned above. He considered how to estimate quantities of the form $E\left(g\left(\Psi_{t}\right) \mid w_{t}\right)$ where $g$ is a Borel function on $R^{1}$ such that $E\left|g\left(\psi_{t}\right)\right|<\infty$ and $w_{t}$ contains a finite number of lags of $\psi_{t}$. Robinson, and the others referred to above, employ the kernal method of moment estimation pioneered by Nadaraya (1964) and Watson (1964) to produce an estimator of the conditional mean of $\psi_{t}$ at $w_{t}=\bar{w}_{t}$ of the form

$$
\hat{E}\left(g\left(\psi_{t}\right) \mid \bar{w}_{t}\right)=\frac{\left[\left(\gamma_{T}^{q} T\right)^{-1} \sum_{j=1}^{T} g\left(\bar{\psi}_{j}\right) K\left(\left(\bar{w}_{t}-\bar{w}_{j}\right) / \gamma_{T}\right)\right]}{\left[\left(\gamma_{T}^{q} T\right)^{-1} \sum_{j=1}^{T} K\left(\left(\bar{w}_{t}-\bar{w}_{j}\right) / \gamma_{T}\right)\right]} .
$$


where $\bar{w}_{j}, \bar{\psi}_{j}$ are observed data, $\gamma_{T}$ is a "bandwidth" parameter that is typically proportional to $T^{-1(4+q)}$, and $K($ ) is a kernal function that aims to smooth the density. Many types of kernals might be employed. A popular one used later in Section 5 is the normal kernal (see Singhand Ullah (1985. p.31) for this).

Under various restrictions upon $\gamma_{T}$, differentiability of the density function of $\psi_{t}, w_{t}$, boundedness of $g\left(\psi_{t}\right)$, and assuming $\left(\psi_{t}, w_{t}\right)$ are strictly stationary stochastic processes that are $\alpha$-mixing with mixing coefficients $\alpha_{j}$ such that $T \sum_{j=T}^{\infty} \alpha_{j}$ is $o(1)$, Robinson shows that $\hat{E}\left(\left.g\left(\psi_{t}\right)\right|_{w_{t}}\right) \stackrel{p}{\rightarrow} E\left(\left.g\left(\psi_{t}\right)\right|_{w_{t}}\right)$ and $\left(a_{T} T\right)^{1 / 2}\left(\hat{E}\left(g\left(\psi_{t}\right) \mid \bar{w}_{t}\right)-E\left(g\left(\psi_{t}\right) \mid \bar{w}_{t}\right)\right)$ has a limiting normal distribution, where $a_{T}=r_{T}^{q}$. If $g\left(\psi_{t}\right)$ is not bounded but has bounded moments of order $h$, Robinson also showed that the normality result goes through provided $T \sum_{j=T}^{\infty} \alpha_{j} 1-(2 / h)$ is $O(1) .(h>2)$. Hence it is possible to estimate the conditional mean and variance of $\psi_{t}$ without specifying the exact way in which the conditional moments depend on $w_{t}$. As well, the central limit theorem enables confidence bands to be placed around estimates.

A number of quantities can be constructed from this result. Suppose non-parametric estimates of $E\left(\left.\psi_{t}\right|_{t}\right)$ and $E\left(\psi_{t}^{2} \mid \bar{w}_{t}\right)$ are available. Then we can define $\hat{\sigma}_{t}^{2}=\hat{E}\left(\psi_{t}^{2} \mid \bar{w}_{t}\right)-\left[\hat{E}\left(\psi_{t} \mid \bar{w}_{t}\right)\right]^{2}$ and thereby derive a consistent estimate of $\sigma_{t}^{2}$ by non-parametric methods. The non-parametric mean estimator $\hat{m}_{t}=\hat{E}\left(\psi_{t} \mid \bar{w}_{t}\right)$ yields non-parametric residuals $\hat{\phi}_{t}=\psi_{t}-\hat{m}_{t}$. Previously such estimates were utilized to set up an IV estimator, and it is natural to do that again. Thus the equation to be estimated is: 


$$
y_{t}=\bar{x}_{t} \gamma+\hat{\phi}_{t}^{2} \delta+e_{t}+\delta\left(\sigma_{t}^{2}-\phi_{t}^{2}\right)+\delta\left(\phi_{t}^{2}-\hat{\phi}_{t}^{2}\right)
$$

wi th instruments $z_{t}$. Proposition 5 gives a statement of conditions under which the IV estimator of $\beta^{\prime}=\left(r^{\prime} \delta\right)$ is consistent when $\hat{m}_{t}$ is found from a non-parametric estimation method.

Proposition 5: Under (i) the conditions set out in Proposition 1,and. (ii) that $\hat{m}_{t}-m_{t}$ is uniformly consistent, i.e. ${\underset{w}{w \in R}}^{\sup _{(}}\left(\hat{m}_{t}-m_{t}\right)$ is $o_{p}(1)$. $\tilde{\beta}=\left(Z^{\prime} X\right)^{-1} Z \cdot y$ is a consistent estimator of $\beta$.

Proof: It is only necessary to show that $T^{-1} \Sigma z_{t}\left(\phi_{t}^{2}-\hat{\phi}_{t}^{2}\right) \stackrel{p}{\rightarrow} 0$ as $T^{-1} \Sigma z_{t} e_{t}$ and $T^{-1} \Sigma z_{t}\left(\sigma_{t}^{2}-\phi_{t}^{2}\right)$ were shown to converge to zero in the proof of Proposition 1 . A sufficient condition for this is that $T^{-1} \Sigma z_{t}\left(\phi_{t}^{2}-\hat{\phi}_{t}^{2}\right) \stackrel{p}{\rightarrow} 0$ or $-2 T^{-1} \Sigma z_{i t} \phi_{t}\left(\hat{\phi}_{t}\right.$ $\left.-\phi_{t}\right)+T^{-1} \sum z_{i t}\left(\hat{\phi}_{t}-\phi_{t}\right)^{2} \stackrel{p}{\rightarrow} 0 \forall i=1, \ldots p$. As the first element in this expression is of higher order than the second we concentrate upon it. re-writing it as $T^{-1} \Sigma z_{i t} \phi_{t}\left(m_{t}-\hat{m}_{t}\right)$. A sufficient condition for $T^{-1} \Sigma z_{i t} \phi_{t}\left(m_{t}\right.$ $\left.-\hat{m}_{t}\right) \stackrel{p}{\rightarrow} 0$ is that $T^{-1} \Sigma\left|z_{i t} \phi_{t}\right|\left|m_{t}-\hat{m}_{t}\right| \stackrel{p}{\rightarrow} 0$, and the latter is bounded by $\left[T^{-1} \Sigma\left|z_{i t} \phi_{t}\right|\right] \stackrel{\sup _{w} \in R^{q}}{ }\left|\hat{m}_{t}-m_{t}\right|$. As $\sup \left|\left(m_{t}-\hat{m}_{t}\right)\right|$ is $o_{p}(1) \cdot T^{-1} \Sigma z_{1 t} \phi_{t}\left(m_{t}-\hat{m}_{t}\right)$ is $o_{p}(1)$ if $T^{-1} \Sigma\left|z_{i t} \phi_{t}\right|$ is $o_{p}(1)$. Because $z_{i t}$ and $\phi_{t}$ are stationary and ergodic processes, from the ergodic theorem (White (1984. Theorem 3.34. p.42)). $T^{-1} \Sigma\left|z_{i t} \phi_{t}\right| \stackrel{p}{\rightarrow} E\left(\left|z_{i t} \phi_{t}\right|\right)$ if $E\left|z_{i t} \phi_{t}\right|<\infty$. But from the Cauchy-Schwartz inequality $E\left|z_{i t} \phi_{t}\right| \leq\left[E\left(z_{i t}^{2}\right) E\left(\phi_{t}^{2}\right)\right]^{1 / 2}<\infty$ under the assumptions of Proposition 1. 
Of course, the pertinent question concerns the likelihood of uniform convergence of $\hat{m}_{t}$. This has been shown under a number of different assumptions about the way in which $\psi_{t}$ and $w_{t}$ are generated. Bierens (1983) allows the processes to be $\phi$-mixing or to have a $\phi$-mixing base and shows uniform consistency under the condition that $\operatorname{Tr}_{T}^{2 q_{i}} \sum_{j=0}^{T} \phi_{j}^{1 / 2} \rightarrow \infty$ as $T \rightarrow \infty$. where $\phi_{j}$ are here the mixing coefficients. Sufficient conditions for this areTr ${ }_{T}^{2 q_{i}}$ $\rightarrow \infty$ and $\sum_{0}^{\infty} \phi_{j}^{1 / 2}<\infty$. When processes are $\alpha$-mixing, using the proof in Singh and Ul lah (1987), $\hat{m}_{t}-m_{t}$ can be shown to be uniformly consistent provided $T_{T}{ }^{2 q} \rightarrow$ $\infty$ and $\sum_{j=0}^{\infty} \alpha_{j}^{1-(2 / h)}<\infty$, where the $h^{\prime}$ th moment of $\dot{\psi}_{T}^{\prime}(h>2)$ is bounded. ${ }^{3}$ Thus uniform convergence of $\hat{m}_{t}$ seems a reasonable assumption for most time series. al though there is no completely general treatment yet available in the li terature.

It is instructive to compare this outcome with that when the residuals $\hat{\phi}_{t}$ derive from a parametric regression. In that case the convergence to zero was at the rate $\mathrm{T}^{-1 / 2}$, whereas sup $\left|\hat{m}_{t}-m_{t}\right|$ normally converges to zero at a rate $\mathrm{T}^{-1 / 2} \mathrm{a}_{\mathrm{T}}^{-1}$, and so the use of non-parametric procedures slows the rate at which consistency is achieved by an amount directly dependent upon the dimension of $w_{t}$. i.e. q. This conclusion supports the intuition that a shift to non-parametric methods requires larger numbers of observations if they are to be successfully employed.

${ }^{3}$ This correction to Singh and Ullah's (1985) result on uniform consistency was suggested by $P$. M. Robinson in a personal communication to the second author. who is grateful for this information. 
Having established consistency under fairly general conditions, questions of inference come to the fore, and these prove to be far more complex. One important case can be dispatched immediately: when it is desired to test if $\delta=0$. A good deal of econometric work involves testing such an hypothesis. i.e. checking for the existence of a risk premium, giving Proposition 6 below a substantial range of application.

Proposition 6: Under the same conditions as in Propositions 2 and 5, and $\delta=0$. the covariance matrix of the IV estimator, formed by generating $\hat{\phi}_{t}$ non-parametrically, is the same as that of Proposition 1, and may be consistently estimated as in Proposition 2.

Proof: When $\delta=0$ the error in $(10)$ is just $e_{t}$, and $i t$ is only necessary to show that $T^{-1} Z \cdot \hat{x}$ is $O_{p}(1)$. The only new term is $T^{-1} \Sigma z_{i t} \hat{\phi}_{t}^{2}$ and this converges to $\mathrm{T}_{\rightarrow \infty} T^{-1} \Sigma z_{i t} \phi_{t}^{2}$ if $T^{-1} \Sigma z_{i t} \phi_{t}\left(\phi_{t}-\hat{\phi}_{t}\right) \stackrel{p}{\rightarrow} 0$. an outcome demonstrated in the proof of Proposition 5.

Unfortunately, once the realm of testing the sharp hypothesis $\delta=0$ is left. it becomes difficult to provide a precise answer to how the covariance matrix of the IV estimator of Proposition 4 should be computed. 4 Ideally, one would like to extend Proposition 3, but this does not seem to be possible. The situation is akin to that in Carroll (1982) in that it requires $T^{-1 / 2} \Sigma z_{t} e_{t}\left(m_{t}-\hat{m}_{t}\right) \stackrel{p}{\rightarrow} 0$. Carroll demonstrated this when all data was

${ }^{4}$ Note that if all one was interested in was testing if $\delta=0$ any proxy for $\sigma_{t}^{2}$ could be entered into a regression of $y_{t}$ against $\bar{x}_{t}$ and the proxy and the OLS results used to test if $\delta=0$. Presumably the power of the test depends upon the correlation of the proxy and $\sigma_{t}^{2}$, and so demands a careful choice. 
independently and identically distributed, $\sigma_{t}^{2}$ was a function of only a single variable $w_{t}$, and $w_{t}$ had bounded, compact support. But it seems very hard to generalize this result. Fundamentally, the difficulty in doing so arises from the fact that $\hat{m}_{t}$ is a ratio of random variables and, with standard kernels such as the normal, there is no guarantee that its finite-sample variance exists. Hence, it becomes hard to apply standard limit theorems. Robinson (1986b) has managed to extend Carroll's result by replacing the kernel estimator $m_{t}$ by a nearest-neighbour estimator, and it may be necessary to follow that route here as well. Clearly, this is an area that will repay future research.

\section{Risk as a Regressand}

It is not always the case that the model to be estimated has risk as a regressor. Sometimes information is sought upon the importance of different influences upon the level of risk, in which case it appears as the dependent variable in a regression. Two examples might be cited. The first arises in the debate over whether the variability in inflation was an increasing function of its level or not. Applications exist in which measures of "inflation risk" are related to the level of inflation, for example Hercowitz (1981). Pagan et al (1983) and Engle (1983). A second type comes from the volatility debate initiated by Shiller (1981), which argued that stock market prices fluctuated much more than dividends. Malkiel (1979) and Pindyck (1984) attribute the decline in real stock market values between the mid-1960's and the early 1980's as due to a rise in risk. This could be captured by assuming that $\sigma_{t}^{2}=\sigma^{2}+\alpha D_{t}$, where $D_{t}$ is a dummy variable that is unity after the time 
in which risk is assumed to rise. Alternative hypotheses in the same line are the investigation by Poterba and Summers (1986), which has $\sigma_{t}^{2}$ following an autoregression $\sigma_{t}^{2}=\alpha_{0}+\alpha_{1} \sigma_{t-1}^{2}$. or French et al (1986) who model it as an ARIMA process.

All of these possibilities can be captured in $(11)^{5}$

$$
\sigma_{t}^{2}=\sum_{j=1}^{k} \alpha_{j} \sigma_{t-j}^{2}+\bar{x}_{t} \gamma
$$

which can be converted to a suitable estimating equation

$$
\phi_{t}^{2}=\sum_{j-1}^{k} \alpha_{j} \phi_{t-j}^{2}+\bar{x}_{t} \gamma+\sum_{j=0}^{k} \alpha_{j}\left(\sigma_{t-j}^{2}-\phi_{t-j}^{2}\right),\left(\alpha_{0}=-1\right)
$$

provided that $\phi_{t}$ exhibits the weak property. It is the divergence between $\phi_{t-j}^{2}$ and $\sigma_{t-j}^{2}$ that makes (12) a stochastic relation.

If the $\alpha_{j}$ in (11) were all zero, (12) would be a standard linear model to which least squares could be applied provided $\bar{x}_{t}$ appeared in the information set: if it did not an instrumental variables estimator would need to be invoked. Thus a consistent estimator of $\boldsymbol{\gamma}$ would be readily available, and the only complication is the need to allow for the heterogeneity in the error term when performing inferences.

\footnotetext{
${ }^{5}$ Poterba and Summers add a disturbance term to (11). Although this just augments the error term in (12) and does not change our argument in any way. it is not at all clear why they should add an error term to (11). By definition $\sigma_{t}^{2}$ is a conditional expectation and therefore a function of variables entering the conditioning set. It would therefore be necessary to argue that the appended error appears in the conditioning set used by agents but is absent from that of the econometrician's.
} 
When lagged terms of $\phi_{t}^{2}$ appear in (12), OLS would generally be inconsistent. Supose that $\bar{x}_{t}$ is absent from (12) and that $k=1$. Then the OLS estimator of $\alpha_{1}$ is $\left.\hat{\alpha}_{1}=\alpha_{1}+\underset{t=1}{T} \phi_{t-1}^{4}\right)^{-1}\left(\sum_{t=1}^{T} \phi_{t-1}^{2}\left(\sigma_{t-1}^{2}-\phi_{t-1}^{2}\right)\right) \alpha_{1}$, and the inconsistency is $\alpha_{1} \mu_{4}^{-1}\left(v-\mu_{4}\right)$, where $v=\lim _{T \rightarrow \infty} T^{-1} \underset{t=1}{T} E\left(\sigma_{t-1}^{4}\right)$ and $\mu_{4}=\underset{T \rightarrow \infty}{\lim }$ $T^{-1} \sum_{t=1}^{T} E\left(\phi_{t-1}^{4}\right)$. If $\phi_{t}$ was conditionally normally distributed then $E\left(\phi_{t}^{4}\right)=E\left[E\left(\phi_{t}^{4} \mid F_{t}\right)\right]=E\left[3 \sigma_{t}^{4}\right]$ and $\mu_{4}=\lim _{T \rightarrow \infty} T^{-1} \sum_{t=1}^{T} 3 E\left(\sigma_{t-1}^{4}\right)$, making the inconsistency equal to $-(2 / 3) \alpha_{1}$. Hence, even if the true value of $\alpha_{1}$ is close to unity, the OLS estimate will tend to be very low by comparison. 6

Estimation of (12) therefore needs to be done by IV. However, the presence of an MA(k) in the disturbance term of (12) introduces a new complication, since al though members of $\mathscr{F}_{t}$ are uncorrelated with $\left(\phi_{t}^{2}-\sigma_{t}^{2}\right)$. they need not be with $\left\{\phi_{t-j}^{2}-\sigma_{t-j}^{2}\right\}_{j=1}^{k}$. What is certain, however, is that elements of $F_{t-k-1}$ will be, and this means that instruments need to be constructed by lagging the information set at least $(k+1)$ times. Of course, this is an old strategy for getting consistent estimators of the AR part of ARMA models.

6 Poterba and Sumners (1986) conclude (p.1147) that the inconsistency is small. despite the fact that they assume normality for the $\phi_{t}$ (footnote $10, p .1147$ ). 


\section{The Properties of Al ternative Risk Measures}

As mentioned in the introduction, there are quite a number of papers that have been concerned to calibrate the effects of risk and have entered some measure of it into regressions. Because these measures are widespread it is important to understand why they can be defective, and we turn to an investigation of that here. Some of these procedures are specific to inflation risk, al though they might be adapted to other contexts as well. Four methods are singled out for analysis.

\section{(a) Moving Average Measures}

This is by far the most popular technique; references would be legion but a small sample is Gylfason (1981). Klein (1977) Ibrahim and Williams (1978) and Pindyck (1984). The strategy here is replace $\sigma_{t}^{2}$ by $\phi_{t}^{2}$ constructed as

$$
\phi_{t}^{2}=m^{-1} \sum_{k=0}^{m-1}\left(\psi_{t-k}-\mu_{t-k}\right)^{2} \text { where } \mu_{t}=m^{-1} \sum_{l=0}^{m-1} \psi_{t-l}
$$

It is not entirely clear what argument for this definition is. Moreover, it is not hard to construct cases where it can be very misleading. For example, suppose that $\psi_{t}=b t+d t^{2}$, and is therefore perfectly predictable. In such circumstances $\sigma_{t}^{2}$ must be zero, and any risk measure should reflect this. But $\phi_{t}^{2}$ as defined above varies systematically with $t$ whenever $m>1$.

The origin of these difficulties is the failure to fully specify the information set underlying the construction of $\sigma_{t}^{2}$. One might at tempt to surmount them by defining $\mathcal{F}_{t}$ as the elements making up $\mu_{t}$, al though that would 
force the moving average to be based only upon past values of $\psi_{t}$. With this proviso, it is apparent that the proposed definition is motivated by the formula for the sample variance of a stationary series (see Klein (p.700)); for non-stationary series it is a misleading indicator of $\sigma_{t}^{2}$, producing at best an average of the $\sigma_{t}^{2}$ 's as $m \rightarrow \infty$. Consequently, variance changes tend to get blurred.

As a regressor, the use of $\phi_{t}^{2}=m^{-1} \sum_{j=0}^{m-1}\left(\psi_{t-j}-\mu_{t-j}\right)^{2}$ in place of $\sigma_{t}^{2}$ must lead to inconsistent estimators if the current value of $\psi_{t}$ appears in it. Few authors appreciate this point: Huizinga and Mishkin (1985) being an exception. One of the few arguments that might be advanced in support of $\phi_{t}^{2}$ as a regressor is that (1) should have $m^{-1} \sum_{j=0}^{m-1} \delta_{t-j}^{2}$ in place of $\delta_{t}^{2}$. Then $\phi_{t}^{2}$ has the strong property that $\phi_{t}^{2} \stackrel{p}{\rightarrow} m^{-1} \sum_{j=0}^{m-1} \sigma_{t-j}^{2}$ as $m \rightarrow \infty$, and, with $m / T \rightarrow 0$ as $T \rightarrow \infty, \delta$ would be consistently estimated by regression. However this would not seem to be what investigators using this procedure actually had in mind, as they almost invariably write the risk terms with no lags.

\section{(b) Relative Price Measures}

One of the most common measures of $\sigma_{t}^{2}$ when it relates to prices or inflation is that derived from a series showing the relationship of individual prices to the aggregate level price. With $P_{i}$ the $\log$ of the prices of the $i^{\text {th }}$ commodity and $P$ the $\log$ of the general price level, $\sigma_{t}^{2}$ is replaced by $\phi_{t}^{2}=$ $m^{-1} \Sigma\left(P_{i t}-P_{t}\right)^{2}$. where the summation is over $m$ commodities. To analyze the utility of this formula, some model of $P_{i t}-P_{t}$ is necessary; the one adopted 
here being that of Lucas (1973) as formulated by Cukierman and Wachtel (1979) and generalized in Pagan et al. (1983). In the latter paper it was shown that, as $m \rightarrow \infty, P_{i t} P_{t}=D_{t} a_{i}+\eta_{t} b_{i}+v_{i t}+o_{p}(1)$, where $\eta_{t}$ is a macroeconomic shock common to all markets, $v_{i t}$ is a market-specific shock and $D_{t}$ represents the mean value of non-price variables shifting demand and supply curves. Cukierman and Wachtel's formulation has $P_{1 t}-P_{t}=v_{i t}+o_{p}(1)$, as they assume $D_{t}=0$ and that all markets identical making $b_{i}=0$.

Wi th the identical market assumption and no systematic factors, $m^{-1} \Sigma\left(P_{i t}-P_{t}\right)^{2}=m^{-1} \Sigma v_{i t}^{2}=\phi_{t}^{2}$ and it is clear that $\phi_{t}^{2} \rightarrow \sigma_{t}^{2}$ as $m \rightarrow \infty$ if $\sigma_{t}^{2}=$ $E\left(v_{i t}^{2}\right)$. Therefore, the desirable strong property of a good variance measure alluded to earlier holds, and OLS delivers consistent estimators. 7 Undoubtedly, it is this type of argument which underlines the popularity of such a measure. It should be clear however, that the strong property fails to hold when markets are not identical. Then the macroeconomic shock common to all has differential effects upon the prices in each market, the diversity being dependent upon the demand and supply responses. Only the weak property for $\phi_{t}^{2}$ can be invoked in the general case, and thus the estimation difficulties outlined in Sections 2 and 3 recur.

\section{(c) Survey Measures}

Sometimes measures of uncertainty due to inflation or interest rate fluctuations have been derived from the dispersion of responses given by

\footnotetext{
7 Note that many studies actually use the inflation rate rather than the price level so that $\phi_{t}^{2} \rightarrow 2 E\left(v_{i t}^{2}\right)$. This may raise questions concerning the definition of $\sigma_{t}^{2}$.
} 
individuals to questions about their anticipations, e.g. Levi and Makin (1979). Analyzed in the context of the same model as that used to establish the characteristics of relative price measures, the survey based approaches are found to have similar deficiencies. This is most clearly seen by examining the polar case where each individual transacts in only one market. 8 so that his anticipated price level combines information from the $1^{\text {th }}$ market with a "macro" projection of the aggregate price level. Designating this by $\hat{P}_{t}(1)$, it was show in in Pagan et al (1983) that $\hat{P}_{t}(i)-P_{t}=\theta_{i}\left(\eta_{t} \beta_{i}+\right.$ $\left.\epsilon_{i t}\right)-\eta_{t} \sum w_{k} \beta_{k}-\Sigma w_{k} \epsilon_{k t}$. where $\theta_{i}$ is the weight given to the $i^{\text {th }}$ price in forming the aggregate anticipation. $w_{k}$ are the weights used in constructing the aggregate price level, and $\beta_{i}$ depends on the demand and supply curve parameters in the $i^{\text {th }}$ market. It is apparent from this formula that the macro shock appears in the survey measure just as it does in indices constructed from relative price dispersion, and therefore the two procedures share the same set of difficulties. An alternative definition is to relate $\hat{P}_{t}(i)$ to $\sum w_{k} \hat{P}_{t}(i)$. i.e.. to the average of respondents' anticipations, in which case $\hat{p}_{t}(i)-\Sigma w_{k} \hat{p}_{t}(i)=\theta_{i}\left(\eta_{t} \beta_{i}+\epsilon_{i}\right)-\Sigma \theta_{k}\left(\eta_{t} \beta_{k}+\exists_{k}\right)$, and the macro shock disappears only if all markets are identical. When markets differ, the macro shock has different effects upon the anticipations of actors, depending upon which market they transact in.

(d) Measures Based on Particular Parameterisations

All of the above methods could be construed as attempts to avoid an explicit parameterisation for $\sigma_{t}^{2}$. Some authors have gone much further

8 Nothing in the argument below depends on this assumption, but the formula given for the price differential will have different weights at tached to the shocks $\eta_{t}$ and $\epsilon_{i t}$. 
however, and explicitly parameterised $\sigma_{t}^{2}$, e.g. Hansen and Hodrick (1983), Domowitz and Hakkio (1984) and Engle et al (1984). What differentiates these studies are the variables $\sigma_{t}^{2}$ is assumed to depend upon and the estimation methods: employed to obtain estimates of the parameters of interest, $\gamma$ and $\delta$. We therefore turn to an examination of some of the main features of this li terature.

Existing studies invariably assume that $\sigma_{t}^{2}$ is linearly related to some variable $z_{t}$ in the form $\sigma_{t}^{2}=\sigma^{2}+z_{t} \alpha$. If observations on $\phi_{t}^{2}$ are available one could re-write this as

$$
\phi_{t}^{2}=\sigma^{2}+z_{t}^{\alpha}+\left(\phi_{t}^{2}-\sigma_{t}^{2}\right)=\sigma^{2}+z_{t} \alpha+v_{t}
$$

and joint estimation of (3) with (13) could be performed. One estimator of (3) and (13) would be the instrumental variables estimator described earlier, but alternative procedures would be to apply FIML or to engage in two-stage procedures (one variant of the latter would be to regress $\phi_{t}^{2}$ against unity and $z_{t}$. producing $\tilde{\sigma}^{2}$ and $\tilde{\alpha}$, and then use $\tilde{\sigma}_{t}^{2}=\tilde{\sigma}^{2}+z_{t} \tilde{\alpha}$ as a regressor in (3)). Two-stage procedures will only provide valid inferences provided the adjustments to the covariance matrix described in Pagan (1984, p. 240) are done, whereas FIML does not share this problem. However. FIML is far more sensitive to specification errors than the instrumental variables estimator. If the set of variables driving (13) is larger than $z_{t}$. FIML will generally be inconsistent, whereas (IV) will not be. Thus, even if $\sigma_{t}^{2}$ is parameterised, there are sound reasons for employing the instrumental variable estimators discussed in Section 2.

Donowitz and Hakkio (1985) examine risk premia in the exchange rate. In their theoretical model $\psi_{t}$ represents foreign and domestic monies. which they take as following a VAR, so that $\hat{\phi}_{t}$ would be the residuals from the fitted 
VAR, and estimation could be done as described above. However, in practice they depart from their theoretical model, making $\sigma_{t}^{2}$ a function of the past history of $e_{t}$, as defined in (3): in particular they force $\sigma_{t}^{2}$ to be Engle's (1982) ARCH process, say $\sigma_{t}^{2}=\sigma^{2}+e_{t-1}^{2} a$, and then apply ULE to (3) to obtain estimates of $\gamma, \beta$ and $\alpha$.

A potential problem with modelling $\sigma_{t}^{2}$ as an ARCH process is that the MLE of $\delta$ is almost certain to be inconsistent if $\sigma_{t}^{2}$ is not of the ARCH form, since the conditional mean in (1) is then mis-specified. Unfortunately, it is not possible to derive an estimate of $\phi_{t}$ (in this case $e_{t}$ ) that could be used to define the IV estimator of section 2 if $\sigma_{t}^{2}$ really is an ARCH process; the reason being that ${ }^{g} t$ would be a function of the entire past history of $\left\{y_{t}, \bar{x}_{t+1}\right\}$, and so non-parametric methods which require $\mathscr{F}_{t}$ to be finite dimensional cannot be applied. If only a finite number of members of $\mathscr{F}_{t}$ are used. $\S_{t}$. Proposition 4 shows that the IV estimator using $\hat{\phi}_{t}^{2}$ based upon $\left(\Psi_{t}-\right.$ $\left.E\left(\Psi_{t} \mid \xi_{t}\right)\right)^{2}$ would be inconsistent.

Faced with this difficulty it is absolutely imperative that some indication of whether the ARCH assumption is valid be obtained. A specification test of the Hausman (1978) type may be implemented in the following way. Let $\hat{e}_{t}$ be the fitted residuals from the ARCH process and $\hat{\sigma}_{t}^{2}$ be the estimated value of $\sigma_{t}^{2}$ implied by the MLE. Form IV estimators of $\delta$ and $r$ in (3) by using $\hat{\sigma}_{t}^{2}$ as an instrument for $\hat{e}_{t}^{2}\left(\hat{e}_{t}=\hat{\phi}_{t}\right.$ in this case). When the $\sigma_{t}^{2}$ do follow an ARCH process it is obvious that this IV estimator is consistent, since $\hat{\sigma}_{t}^{2}$ is a function of past information and this has zero correlation with $e_{t}^{2}-\sigma_{t}^{2}$. In contrast, the most efficient estimator of $\delta$ and $r$ under these conditions is the MLE. A comparision of these two estimators is 
therefore the suggested specification error test. Provided the specification is correct both estimators are consistent, whereas under the al ternative there is no reason to believe that they will have the same probability limit since the set of first order conditions defining both estimators are quite different. Precisely in what circumstances this test will be powerful is however beyond this paper.

\section{Two Applications}

\subsection{Risk in the Foreign Exchange Narket}

The presence of a risk premium in foreign exchange markets has been much debated. A number of studies have concluded that there is a risk premium, but that the evidence for it is fairly weak, e.g. Hansen and Hodrick (1983) and Domowitz and Hakkio (1985). Many studies of the efficiency of the foreign exchange market actually proceed as if there were no risk premium, and a good example of this is the research reported by Longworth (1981) on the Canadian-U.S. exchange market over the period $1970 / 7$ to $1978 / 12$. As the data used in that study was available to us, we undertook to examine the evidence for a risk premium in that market over the sample period.

The data for the model constituted the $\log$ of the spot rate $\left(S_{t}\right)$ and the $\log$ of the 30-day forward rate $F_{t-1}$. Longworth fitted the standard model $(\delta=0)$

$$
S_{t}=a+b F_{t-1}+\delta \sigma_{t}^{2}
$$

and tested market efficiency with the null hypothesis $a=0, b=1$. In the first column of Table 1 we present results from this regression; estimates of a and $\mathrm{b}$ are close to their theoretical values. However, a time series analysis of 
$S_{t}$ and $F_{t}$. shows that both series are $\operatorname{ARIMA}(0,1,0)$ with no evidence of any drift, and therefore the "t statistics" in Table 1 cannot be taken to be asymphotetically normally distributed (Phillips and Durlauf (1986)). A close examination of the residuals of the estimated model points to

TABLE 1: Estimated Model of the $\$ U S / \$ C^{*}$

$\begin{array}{llcc}\text { a } & (1) & (2) & (3) \\ \text { b } & .00036 & -.0013 & -.0008 \\ & (.29) & (1.19) & (.65) \\ & 1.0229 & 1.0291 & 1.0276 \\ & (43.29) & (52.90) & (51.72) \\ & & 19.311 & 13.975 \\ & & (6.71) & (2.20)\end{array}$

* The estimated model is $s_{t}=a+b F_{t-1}+\delta \sigma_{t}^{2}$. In (2) the proxy for $\sigma_{t}^{2}$ is $\hat{\phi}_{t}^{2}$. where $\hat{\phi}_{t}$ is generated non-parametrically with conditioning set $F_{t}=$ $\left[S_{t-1}-F_{t-2}, S_{t-2}-F_{t-3}\right]$. In (3) estimation is by generalized instrumental variables with the non-parametric estimator of $\sigma^{2}$ used as instrument for $\hat{\phi}_{t}^{2}$. Non-parametric estimation was performed as described in the text. Absolute "t-values" are in parentheses. All computations were done in the DFIT micro-computar package written by B. and H. Pesaran on a COMPAQ Deskpro 286.

potential weaknesses in it. In particular there is evidence of non-linear effects, with the t-statistic for the regression of the squared residuals against their lagged value being 2.05 .

Our first task is to check for the existence of a risk premium, i.e. to test if $\delta=0$ in (14). As observed in footnote 3, any proxy for $\sigma_{t}^{2}$ could be used for that purpose. Moreover. OLS is a perfectly appropriate estimator of 
$\delta=0$ since, under the null hypothesis, the correlation of $\phi_{t}^{2}$ with $\delta\left(\sigma_{t}^{2}-\phi_{t}^{2}\right)$ is zero. The best proxy to use would be $\sigma_{t}^{2}$. On the basis of theoretical models such as Stockman (1978), measures of $\sigma_{t}^{2}$ should derive from the conditional variances of domestic and foreign money supplies. Instead we follow Domowitz and Hakkio (1985) who make $\phi_{t}=e_{t}=s_{t}-F_{t-1}-\delta \sigma_{t}^{2}=\psi_{t}-E\left(\psi_{t} \mid F_{t}\right)$. where $\psi_{t}$ $=S_{t}-F_{t-1} \cdot{ }^{9} F_{t}$ was then defined as $) \Psi_{t-1} \cdot \Psi_{t-2}$ ) and a non-parametric estimate of $\phi_{t}$ was found. The normal kernal, $\kappa(\cdot)=(2 \pi)^{-1 / 2} \exp \left\{-\left(2 \gamma_{T}^{2} s_{\Psi}^{2}\right)^{-1}\left[\left(\psi_{t-1}-\bar{\Psi}_{t-1}\right)^{2}+\left(\Psi_{t-2}-\bar{\psi}_{t-2}\right)^{2}\right]\right\}$. where $s_{\Psi}$ is $t j e$ sample standard deviation of $\Psi_{t} \cdot \bar{\Psi}_{t-j}$ is the observed value of $\Psi_{t-j}$ and $\gamma_{t}=$ $T^{-1 / 6}$, was used to compute $E\left(\psi_{t} \mid \bar{\psi}_{t-1}, \bar{\psi}_{t-2}\right)$ (arguments for the use of $s_{\Psi}$ and ${ }_{T}$ of this form are given in Singh and Ullah (1985)). $\hat{\phi}_{t}^{2}$ was then used as a proxy for $\sigma_{t}^{2}$, and the resulting regression is given in column 2 of Table 1 . Proposition 6 covers this case.

From the results in Table 1 there is very strong evidence that the basic "efficiency" model is not a complete representation of the data. Of course. rejection of the null hypothesis $\delta=0$ need not lead to acceptance of the alternative that the missing factor is a risk premium, but the fact that theoretical models accord it a role in the determination of the discount $S_{t}$ $F_{t-1}$ is suggestive. It is of some interest to see how $\sigma_{t}^{2}$ varied over the sample period. Accordingly, $\sigma_{t}^{2}$ was estimated non-parametrically in the same

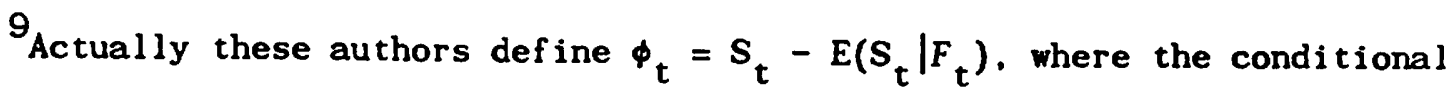
expectation is $a+b F F_{t-1}+\delta \sigma_{t}^{2}$ and $\sigma_{t}^{2}$ is defined as an ARCH process in terms of $\phi_{t-j}^{2}$. Because $S_{t}$ and $F_{t}$ are $\operatorname{ARIMA}(0,1,0)$, we assume that the co-integrating vector is $(1-1)$ as given by theory. This must also be true for Domowitz and Hakkio, as the asymptotic theory for ARCH processes requires $\phi_{t}$ to be stationary and this could not be so if $b \neq 1$. 
way as $\phi_{t}$ was, and figure 1 provides a graph of that series. What is most evident is the rise in $\sigma_{t}^{2}$ that occurs in 1976; the most likely explanation being the increase in uncertainty over this period due to the election of the Parti Quebeiois, whose platform emphasized the separation of Quebec from Canada.

Column three of Table 1 estimates $\delta$ by the instrumental variables estimator of Proposition 5, where the instrument for $\hat{\phi}_{t}^{2}$ is effectively $\hat{\sigma}_{t}^{2}$. In fact. Proposition 5 showed that the estimator of $\delta$ would be inconsistent. although no limiting distribution could be found. What is of most interest in this column is that the OLS estimate of $\delta$ actually exceeds the IV estimate. Al though the OLS estimator inconsistency should always be negative because of the "errors in variables" problem, in this instance there is another source of bias arising from the fact that $\hat{\phi}_{t}^{2}=\hat{e}_{t}^{2}$. If the $e_{t}$ in (2) are not symmetrically distributed, the OLS estimator of $\delta$ would be inconsistent due to correlation of regressor with disturbance. The inconsistency in the OLS estimator due to this effect is positive if $E\left(e_{t}^{3}\right)$ is positive, and can easily outweigh the errors in variables bias if $E\left(e_{t}^{3}\right)-E\left(e_{t}^{4}\right)+\left(E\left(e_{t}^{2}\right)\right)^{2}$ exceeds zero. since the sign of the inconsistency depends on that quantity. In fact. the non-parametric residuals $\hat{\phi}_{t}$ have a positive third moment that is some ten times greater than the fourth moment, and this seems to be the likely explanation for the relative magnitudes of the OLS and IV estimators of $\delta$ in Table 1.

\subsection{Inflation Volatility and Real Effects}

The real effects of high and variable inflation were a much debated issue in the early 1980's and a number of papers investigated the quantitative impact of a volatile inflation rate upon output growth, employment. 


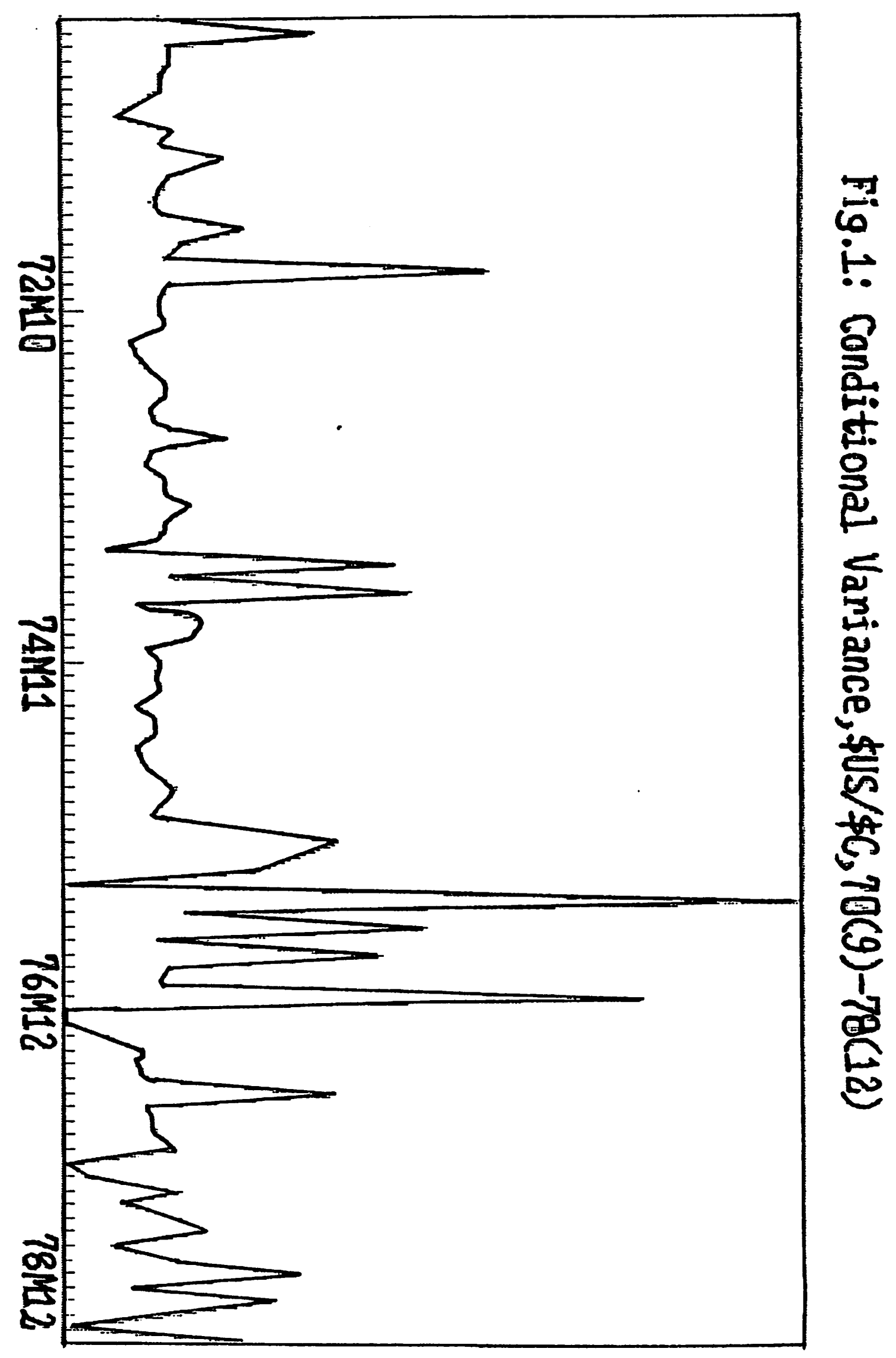


investment, etc. Coulson and Robins (1985) is a good representative of such studies. They argued that the rate of unemployment $\left(R_{t}\right)$ could be dynamically related to the unanticipated inflation rate $\left(\phi_{t}\right)$ and an index of the volatility of inflation $\left(\sigma_{t}^{2}\right)$. Specifically, they estimate the equation

$$
R_{t}=a+\delta \sigma_{t}^{2}+\sum_{j=1}^{8} c_{j} R_{t-j}+\sum_{j=0}^{6} d_{j} \phi_{t-j}
$$

restricting $c_{5}, c_{6}$ and $c_{7}$ to be zero. (15) can be re-parameterized to give $\gamma_{0}$ $=\sum_{j=1}^{8} c_{j}$ and $\gamma_{1}=\sum_{j=0}^{6} d_{j}$ as coefficients, and with series on $\sigma_{t}^{2}$ and $\phi_{t}(15)$ can be estimated with data over the period $1951 / 1$ to $1979 / 4$.

Coulson and Robins followed Engle (1983) in assuming that the inflation rate $\left(\psi_{t}\right)$ was a function of a number of variables such as the money growth rate $\left(w_{t}\right)$, while $\sigma_{t}^{2}$ followed an ARCH process. Estimates of $\phi_{t}$ and $\sigma_{t}^{2}$ were then made from the residuals, $\hat{\phi}_{t}$, and estimated variances, $\hat{\sigma}_{t}^{2}$, generated af ter maximum likelihood estimation of the ARCH process.

Coulson and Robins regressed $R_{t}$ against a constant, $\hat{\sigma}_{t}^{2}, R_{t-j}$ and $\hat{\phi}_{t-j}$ and found that $\delta$ was -.698 with a t-statistic of -3.772 . To parallel Coulson and Robins' work we took the civilian unemployment series from Gordon (1984) as $R_{t}$ and values for $\hat{\phi}_{t}$ and $\hat{\sigma}_{t}^{2}$ from Engle (1983). Column 1 of Table 2 gives "Coulson-Robins" estimates. It is apparent that, although the estimate of $\delta$ is different, the same conclusions about the impact of $\sigma_{t}^{2}$ upon $R_{t}$ would be reached as in the earlier study. 10

${ }^{10}$ Actually, because $\hat{\sigma}_{t}^{2}$ is a function of $\hat{\theta}$. the standard errors of $\hat{\delta}$ from a regression package will be biased - Pagan (1984. p.241) - unless account is taken of the fact that $\theta$ is estimated. However. we could make no adjustment for this as the original data Engle used could not be located. 
Column 2 of Table 2 shows what would happen if $\hat{\phi}_{t}^{2}$ was used as a regressor proxying for $\sigma_{t}^{2}$. In fact, it is not unusual in investigations of this sort for the $\hat{\phi}_{t}$ to be the residuals from a fitted ARMA model. As predicted, the resulting estimator of $\delta$ is severely biased, and an investigator doing this regression would inevitably conclude that inflation volatility had negligible effects upon unemployment. Finally, column 3 estimates the equation of column 2 with Sargan's generalized IV estimator, where the list of instruments is the regressors with $\hat{\phi}_{t}^{2}$ replaced by $\hat{\sigma}_{t}^{2}$. Now there is once again evidence of an effect of $\sigma_{t}^{2}$ upon unemployment, emphasizing the importance of instrumenting $\hat{\phi}_{t}^{2}$ when attempting to estimate $\delta$

\section{Table 2}

Estimates of the Parameters of Equation $(15)^{*}$

$\begin{array}{cccc} & (1) & (2) & (3) \\ \mathrm{a} & .3760 & .2943 & .2882 \\ \delta & (2.60) & (2.00) & (1.44) \\ & -.5340 & -.0680 & -.9194 \\ \gamma_{0} & (2.91) & (.75) & (2.06) \\ & .9542 & .9458 & .9848 \\ \gamma_{1} & (35.3) & (33.72) & (22.85) \\ & .6420 & .3017 & 1.1527 \\ & (3.01) & (1.59) & (2.3)\end{array}$

*Absolute values of $t$-statistics in parantheses $\gamma_{0}=\Sigma c_{j} \cdot \gamma_{1}=\Sigma d_{j}$. Data on $R_{t}$ is from Gordon (1984), $\hat{\phi}_{t}$ and $\hat{\sigma}_{t}^{2}$ from Engle (1983). Column (1) is OLS using $\hat{\sigma}_{t}^{2}$ in place of $\sigma_{t}^{2}$. Column (2) is OLS using $\hat{\phi}_{t}^{2}$ in place of $\sigma_{t}^{2}$. Column (3) is GIVE with $\hat{\sigma}_{t}^{2}$ replacing $\hat{\phi}_{t}^{2}$ in the list of instruments. 


\section{Conclusion}

In this paper we have attempted to provide an integrated approach to the estimation of models with risk terms. In sections two and three it was argued that there exists or thogonality conditions between variables in the information set and higher order moments of the unanticipated variable density. These could be exploited to provide consistent estimators of the parameters associated with the risk term. Specifically, it was recommended that an IV estimator should be applied, with instruments constructed from the information set. Viewed in this way, our analysis represents an extension of current methods for the estimation of models featuring anticipations.

Differences largely stem from the fact that higher order moments are involved. but these are sufficiently distinctive to justify a separate treatment.

Section 4 analysed four existing methods commonly used to estimate models with risk terms. These involved the construction of risk moving averages of time series, risk measures from relative prices and survey data, and direct parameterisation. Various problems with the use of each method were identified. Finally, section 5 used the theory of earlier sections to re-examine a few applied studies in the literature. These involved the presence of a risk term in the \$US/\$C exchange market, and the effects of price uncertainty upon production. 


\section{REFERENCES}

Bierens, H. (1983). "Uniform Consistency of Kernel Estimators of a Regression Function under Generalized Conditions," Journal of American Statistical Association, 78, 699-707.

Bierens, H. (1985), "Kernal Estimators of Regression Functions", (paper presented at the Fifth World Congress of the Econometric Society, Boston).

Carroll. R. J. (1982), "Adapting for Heteroscedasticity in Linear Models". Annals of Statistics, 10, 1224-1233.

Coulson, N. E. and R. P. Robins (1985). "Aggregate Economic Activity and the Variability of Inflation: Another Look". Economics Letters, 17, 71-75.

Cukierman, A. and P. Wachtel (1979), "Differential Inflationary Expectations and the Variability of the Rate of Inflation: Theories and Evidence". American Economic Review, 69, 595-609.

Domowitz, I. and C. S. Hakkio (1985), "Conditional Variance and the Risk Premium in the Foreign Exchange Market". Journal of International Economics, 19, 47-66.

Engle, R. F. (1982), "Autoregressive Conditional Heteroscedasticity with Estimates of the Variance of United Kingdom Inflation". Econometrica, 50. 987-1008.

Engle, R. F. (1983), "Estimates of the Variance of U.S. Inflation Based Upon the ARCH Model". Journal of Money. Credit and Banking, 15, 286-301.

Engle, R. F., D. M. Lillien and R. P. Robins (1987), "Estimating Time Varying Risk Premia in the Term Structure: The ARCH-Model". Econometrica, 55, 391-407.

Engle, R. F. and C. W. J. Granger (1987), "Dynamic Model Specification with Equilibrium Constraints: Co-Integration and Error Correction". Econometrica, 55, 251-276.

Frankel, J. (1985). "Portfolio Crowding - Out Empirically Estimated". Quarterly Journal of Economics, Vol C. Supplement, 1041-1065.

French, K.R., G. W. Schwert and R.F. Stambaugh (1987), "Expected Stock Returns and Volatility". Managerial Economics Research Center Discussion Paper 85-10. University of Rochester.

Friedman, M. (1977), "Nobel Lecture: Inflation and Unemployment". Journal of Political Economy, 85, 451-1065.

Gylfason. T. (1981). "Interest Rates, Inflation and the Aggregate Consumption Function". Review of Economics and Statistics, 63, 233-245.

Gordon. R. J. (1984), Macroeconomics (Little, Brown and Co., Boston). 
Hansen, L. P. and R. J. Hodrick (1983). "Risk Averse Speculation in the Forward Foreign Exchange Market: An Econometric Analysis of Linear Models", in J. A. Frenkel (ed.), Exchange Rates and Internationc.l Macroeconomics (Chicago: University of Chicago Press for the National Bureau of Economic Research).

Hausman, J. (1978), "Specification Tests in Econometrics". Econometrica, 46. 1251-1272.

Huizinga, J. and F. S. Mishkin (1985). "Monetary Policy Regime Shifts and the Unusual Behavior of Real Interest Rates". paper given to the Carnegie-Rochester Conference on Public Policy.

Hercowitz, Z. (1981). "Money and the Dispersion of Relative Prices", Journal of Political Economy. 89, 328-356.

Ibrahim, I.B. and R. Williams (1978), "Price Unpredictability and Monetary Standards: A Comment on Klein's Measure of Price Uncertainty", Economic Inquiry, $16,431-437$.

Klein, B. (1977), "The Demand for Quality-Adjusted Cash Balances: Price Uncertainty in the U.S. Demand for Money Function", Journal of Political Economy, 85, 691-715.

Lawrence, C. (1983), "Rational Expectations, Supply Shocks and the Stability of the Inflation-Output Tradeoff". Journal of Monetary Economics, 11.
225-245.

Leland, H. E. (1968), "Saving and Uncertainty: The Precautionary Demand for Saving". Quarterly Journal of Economics, 82, 465-473.

Longworth, D. (1981). "Testing the Efficiency of the Canadian-U.S. Exchange Market Under the Assumption of No Risk Premium". Journal of Finance. 36.
43-49.

Levi, M. and J. Makin (1979), "Fisher. Phillips. Friedman and the Measured Impact of Inflation on Interest". Journal of Finance, 34, 35-52.

Lucas, R. E. (1973), "Some International Evidence on Output-Inflation Tradeoff s", Anerican Economic Review, 63, 326-334.

Malkiel. B. G. (1979), "The Capital Formation Problem in the United States". Journal of Finance, 34, 291-306.

Mascaro, A. and A. H. Meltzer (1983). "Long and Short-Term Interest Rates in a Risky World". Journal of Monetary Economics, 12, 485-518.

Nadaraya. E. A. (1964), "On Estimating Regression". Theory of Probability and its Applications, 9, 141-142.

Nelson, C. R. (1975). "Rational Expectations and the Estimation of Econometric Models". International Economic Review, 16. 555-561. 
Neuey. W. and K. West (1986), "A Simple Positive Definite, Heteroskedasticity and Autocorrelation Consistent Covariance Matrix", N.B.E.R. Technical Paper No. 55.

Pagan, A. R. (1984). "Econometric Issues in the Analysis of Regressions with Generated Regressors", International Economic Review, 25, 221-247.

Pagan, A. R., A. D. Hall and P. K. Trivedi (1983), "Assessing the Variability of Inflation". Review of Economic Studies, 50, 585-596.

Pindyck, R. S. (1984), "Risk, Inflation and the Stock Narket". American Economic Review, 74, 335-351.

Phillips, P. C. B. (1985), "Time Series Regression with Unit Roots", Cowles Foundation Discussion Paper No. 740, Yale University.

Phillips, P.C.B. and S.N. Durlauf (1986), "Multiple Time Series with Integrated Variables", Review of Economic Studies, 53, 473-496.

Poterba, J. M. and L. H. Summers (1986), "The Persistence of Volatility and Stock Market Fluctuations", American Economic Review, 76, 1142-1151.

Robinson, P. M. (1983). "Non-parametric Estimators for Time Series", Journal of Time Series Analysis, 4, 185-207.

Robinson, P. M. (1986a), "Asymptotically Efficient Estimation in the Presence of Heteroscedasticity of Unknown Form" (Mimeo, London School of Economics).

Robinson. P. M. (1986b), "Nonparametric Methods in Specification," Economic Journal, supplement, $96,134-141$.

Sargan, J. D. (1958), "The Estimation of Relationships Using Instrumental Variables", Econometrica, 26, 393-415.

Shiller, R. J. (1981). "Do Stock Prices Move Too Much to be Justified by Subsequent Changes in Dividends?". American Economic Review, 71, 421-436.

Sims, C. A., J. H. Stock and K. W. Watson (1986). "Inference in Linear Time Series Models with Some Unit Roots". (Mimeo, Stanford University).

Singh, R. S. and A. Ullah (1985), "Nonparametric Time Series Estimation of Joint DGP. Conditional DGP and Vector Autoregression". Econometric Theory, 1, 27-52.

Singh.R.S. and A.Ul lah (1987). "Corrections". (mimeo.University of Western Ontario)

Stock, J.H. (1984), "Asymptotic Properties of Least Squares Estimators of Co-Integrating Vectors", mimeo, Harvard University. 
Stockman. A. (1978), "Risk Information and Forward Exchange Rates', in J. A. Frenkel and H. G. Johnson (eds.) The Economics of Exchange Rates, Reading. Kassachusetts: Addison-Tesley, 1978.

Ul lah. A. (1986), "Non-Parametric Estimation of Econometric Functionals", (Mimeo. University of Western Ontario).

Vanderhoff. J. (1983). "Support for Rational Expectations Nodels with U.S. Data". Journal of Monetary Economics, 12, 297-308

Watson, G. S. (1964), "Smooth Regression Analysis", Sankhya, Series A, 26. 359-372.

White, H. (1980), "A Heteroscedasticity-Consistent Covariance Matrix Estimator and a Direct Test for Heteroscedasticity". Econometrica, 48, 817-838.

White H. (1984), Asymptotic Theory for Econometriclans (New York: Academic Press).

Wickens, M. R. (1982), "The Efficient Estimation of Econometric Models with Rational Expectations". Review of Economic Studies, 49, 55-68. 